\title{
Osmolarity influences chondrocyte repair after injury in human articular cartilage
}

Yuelong Huang ${ }^{1+}$, Yujun Zhang ${ }^{2 \dagger}$, Xiaoquan Ding ${ }^{1}$, Songyang Liu ${ }^{1}$ and Tiezheng Sun ${ }^{1 *}$

\begin{abstract}
Background: The purpose was to determine the influence of irrigation solution osmolarity on articular chondrocytes survival and metabolic state following mechanical injury.

Methods: Osteochondral explants were harvested from patients undergoing total knee arthroplasty for osteoarthritis and then cut through their full thickness to establish mechanical injury models. Cartilage explants were incubated in irrigation solutions (saline and balanced salt) with different osmolarities (180, 280, 380, $580 \mathrm{mOsm} / \mathrm{L})$ for $2 \mathrm{~h}$. The percentage of cell death $(100 \times$ number of dead cells/number of dead and live cells $)$ was quantified with the laser confocal microscopy. The terminal deoxynucleotidyl transferase-mediated dUTP nick-end labeling (TUNEL) assay was performed to detect apoptosis index of injured cartilage. The contents of proteoglycan elution were determined by spectrophotometer at $530 \mathrm{~nm}$, and HIF-1a and type II collagen mRNA yields were quantified with real-time PCR.
\end{abstract}

Results: In situ dead chondrocytes were mainly localized to the superficial tangential region of injured cartilage edge after mechanical injury. The percentage of cell death was decreased, and proteoglycan elution was gradually reduced with the increasing of osmolarity. The apoptosis indices of TUNEL assay in different osmolarities had no significant difference $(P=0.158)$. HIF-1a and type II collagen mRNA yields were the least for chondrocytes exposed to $180 \mathrm{mOsm} / \mathrm{L}$ medium and were the greatest for chondrocytes exposed to $380 \mathrm{mOsm} / \mathrm{L}$ medium. Compared with the saline group, the cell death of superficial zone was significantly decreased $(P=0.001)$ and contents of proteoglycan elution were also significantly decreased $(P=0.045)$ in the balanced salt. HIF-1a $(P=0.017)$ and type II collagen $(P=0.034) \mathrm{mRNA}$ yields in the chondrocytes exposed to the balanced salt were significantly more than the saline group.

Conclusion: The osmolarity of irrigation solutions plays an important role in the survival and metabolic state of chondrocytes following mechanical injury, and the chondrocyte death is not caused by apoptosis. Increasing osmolarity of irrigation solutions may be chondroprotective with decreasing the chondrocyte death, reducing inhibition of metabolism and proteoglycan elution, ultimately preventing cartilage degeneration and promoting integrative repair.

Keywords: Osmolarity, Irrigation solution, Chondrocyte, Articular cartilage

\section{Background}

Numerous arthroscopic procedures involve wounding articular cartilage, for instance, during osteochondral harvest for transplantation or debridement of chondral defect [1]. Such surgical injury results in a region of chondrocyte death and extracellular matrix degradation at the wounded edge that limits successful

\footnotetext{
* Correspondence: tiezheng_sun@163.com

${ }^{\dagger}$ Equal contributors

${ }^{1}$ Arthritis Clinic and Research Center, Peking University People's Hospital, No.

11 Xizhimen South Street, Xicheng District, Beijing 100044, China

Full list of author information is available at the end of the article
}

integrative cartilage repair. During articular surgical procedure, synovial fluid is replaced by the irrigation solutions for visualization of the joint, with the osmolarity ranging from 250 to $300 \mathrm{mOsm} / \mathrm{L}$. However, the osmolarity of normal human synovial fluid is approximately $400 \mathrm{mOsm} / \mathrm{L}$ [2]. In situ chondrocytes therefore experience a marked decrease in extracellular osmolarity during the arthroscopic surgery. Nevertheless, in situ chondrocytes are osmotically sensitive and react to change in extracellular osmolarity [3,4]. In an animal model, the osmolarity of the extracellular matrix has a

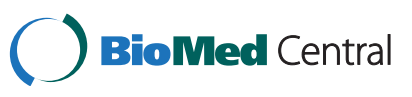

(C) 2015 Huang et al.; licensee BioMed Central. This is an Open Access article distributed under the terms of the Creative Commons Attribution License (http://creativecommons.org/licenses/by/4.0), which permits unrestricted use, distribution, and reproduction in any medium, provided the original work is properly credited. The Creative Commons Public Domain Dedication waiver (http://creativecommons.org/publicdomain/zero/1.0/) applies to the data made available in this article, unless otherwise stated. 
significant impact on chondrocytes following mechanical injury that a decrease in extracellular osmolarity increases chondrocyte death, whereas an increase in extracellular osmolarity has a protective effect [5]. However, only one report from Amin et al. talked about the influence of osmolarity changes on human articular cartilage [6], and the mechanism responsible for influence of the medium osmolarity on the survival of chondrocyte following mechanical injury remains unclear. Modulating the osmolarity to find appropriate properties of irrigation solutions will minimize chondrocyte death from the mechanical injury and be beneficial to retain a viable cell population within the wounded edge, which is favorable of better cartilage repair.

Mechanical injury model was established with human articular cartilage and was incubated in irrigation solutions of different osmolarities for about $2 \mathrm{~h}$, and then, we determined the influence of irrigation solution osmolarity on articular chondrocyte survival, proteoglycan elution, and metabolic state following mechanical injury.

\section{Materials and methods Cartilage preparation}

Human osteochondral materials were obtained from 20 patients undergoing total knee arthroplasty (TKA) for osteoarthritis. The grade 0 or 1 cartilage was selected under macroscopic and microscopic observation, according to International Cartilage Repair Society (ICRS) cartilage tissue scores $[4,7]$. All osteochondral tissues were immediately placed in serum-free Dulbecco's Modified Eagle's Medium (DMEM; Invitrogen Ltd, USA) and were used within $1 \mathrm{~h}$ after resection. The mean age of the patients with osteoarthritis was 69 years old (range: 65-76 years), and there were 3 males and 17 females. Written informed consent for the study was obtained from all of the patients. The research protocol was approved by the Institutional Review Board of the Peking University People's Hospital.

\section{Mechanical injury model}

Human osteochondral tissues from lateral femoral condyle of eight patients were vertically cut through their full thickness with a fresh no. 24 scalpel blade to produce mechanical injury models at the edge of the scalpel cut. The osteochondral tissues were cut into $5 \times 3 \mathrm{~mm}$ rectangular osteochondral explants, consisting of the full-thickness cartilage with subchondral bone (approximately $2 \mathrm{~mm}$ ) (Figure 1). Osteochondral explants were randomly incubated in irrigation solutions with different osmolarities $(180,280,380$, and $580 \mathrm{mOsm} / \mathrm{L}$ ) and primary solutions (saline and balanced salt) at $5 \% \mathrm{CO}_{2}, 37^{\circ} \mathrm{C}$ for $1.5 \mathrm{~h}$. Saline was obtained from China Resources Pharmaceutical Company (National Drug Approval No: H20023300, major ingredients: sodium chloride, $154 \mathrm{mmol} / \mathrm{L}$ ); balanced salt was obtained form Tiancheng Pharmaceutical company (National Drug Approval No: H20130032; major ingredients: sodium chloride, $89 \mathrm{mmol} / \mathrm{L}$; sodium gluconate, $23 \mathrm{mmol} / \mathrm{L}$; potassium chloride, $5 \mathrm{mmol} / \mathrm{L}$; and magnesium chloride, $1.48 \mathrm{mmol} / \mathrm{L}$ ). The osmolarity of the solutions was adjusted to $180,280,380$, and $580 \mathrm{mOsm} / \mathrm{L}$ by adding distilled water or sucrose respectively and measured with a freezing point osmometer (Advanced Micro Osmometer, Model 3300; Vitech Scientific Ltd, UK).

After that, explants were exposed to $10 \mu \mathrm{M}$ fluorescent probes 5-chloromethyl-fluorescein diacetate (CMFDA; Invitrogen, USA) and $10 \mu \mathrm{M}$ propidium iodide (PI; Invitrogen, USA) for additional $30 \mathrm{~min}$ of incubation to label live and dead cells, respectively. CMFDA can freely pass through the cell membrane and produce impermeable 5-chloromethyl-furfural (5-CMF) after an enzymatic reaction, and the green fluorescence is stimulated so that the cytoplasm is colored green that represents the living cells. PI has a positive charge and can only penetrate dead cell membrane and combine with a nucleus that is colored red [8]. Finally, the explants were transferred to $10 \%$ formalin for fixation and stored in $4^{\circ} \mathrm{C}$ phosphate-buffered saline before microscopy.

Using a confocal laser scanning microscope (Leica TCS SP8, Germany), optical sections were acquired in the coronal plane from mechanical injury models (superficial and deep), counting the number of chondrocyte death based on fluorescence and then quantifying the percentage of cell death. The microscope was fitted with $(\times 10)$ objective and obtained the fluorescence channels emitted from CMFDA $(\lambda$ ex $=488 \mathrm{~nm}$ and $\lambda \mathrm{em}=517 \mathrm{~nm})$ and PI $(\lambda \mathrm{ex}=543 \mathrm{~nm}$ and $\lambda \mathrm{em}=650 \mathrm{~nm})$, respectively. By moving the focal plane into the depth of the cut surface, consecutive series of optical sections were acquired at intervals of $10 \mu \mathrm{m}$ in the coronal planes, to a depth of approximately $150 \mu \mathrm{m}$, and finally made the reconstructed three-dimensional image. By moving the focal point to regions of interest, the three-dimensional image was obtained from different coronal planes and the total volume of each region of interest (ROI) for subsequent quantitative analysis was $1,500 \times 600 \times 150 \mu \mathrm{m}^{3}(x-y-z$ axes, respectively). Using Leica QWin Image analysis software (Leica Corporation, Germany), the number of living cells and dead cells was quantified, and then the percentage of cell death was calculated.

\section{TUNEL assay}

The cartilage explants from five patients were obtained using the same procedure as described above and randomly exposed to different osmolarity solutions for $2 \mathrm{~h}$. Terminal deoxynucleotidyl transferase-mediated dUTP nick-end labeling (TUNEL) assay was used to detect apoptotic cells, with a TUNEL Assay Kit (Roche, Germany). 

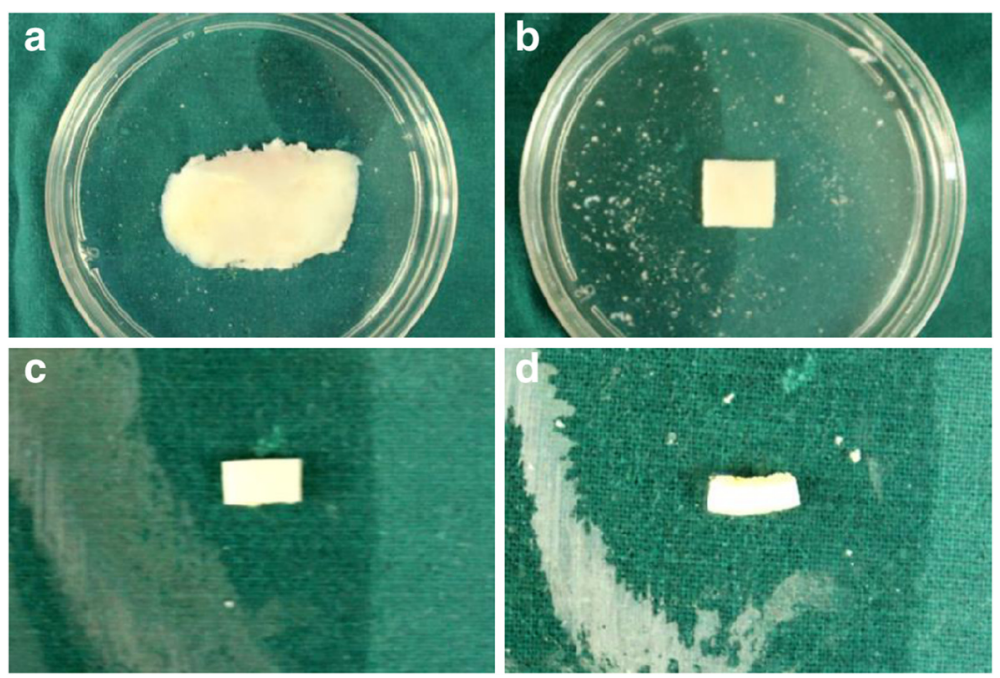

Figure 1 Making the human articular cartilage mechanical injury model. (a) Distal articular surface of femoral lateral condylar of the knee joint. (b) Osteochondral tissue consisting of the full-thickness cartilage with subchondral bone. (c) The osteochondral tissue is vertically cut into explants to produce mechanical injury model at the margin. (d) The lateral view of mechanical injury model on osteochondral explant.

Cells with sepia nucleus were assessed as positive. The percentage of apoptotic cells (apoptosis index) was evaluated by randomly selected five fields $(\times 100)$ in each section from different osmolarity groups.

\section{Proteoglycan elution from cartilage}

The osteochondral explants from lateral femoral condyle of seven patients were obtained using the same procedure as described above and then were randomly incubated at $5 \% \mathrm{CO}_{2}, 37^{\circ} \mathrm{C}$ for a further $2 \mathrm{~h}$ culture in the respective solutions with different osmolarities. An aliquot of the supernatant extracted from the medium was used to determine the contents of proteoglycan elution by measuring the absorbance at $530 \mathrm{~nm}$ on a spectrophotometer using the dimethyl-methylene blue method [9]. The concentration of proteoglycan was measured by the use of chondroitin sulfate to draw a calibration curve.

\section{Primary chondrocytes culture}

Articular cartilage from lateral tibial plateau of fifteen patients was cut into about $1 \mathrm{~mm}^{3}$ fragments and digested with $0.2 \%$ protease (Sigma, USA) for $1 \mathrm{~h}$ and then were further digested with $0.15 \%$ collagenase II (Invitrogen, USA) in DMEM for overnight. Chondrocytes were collected and resuspended in 15\% FBS-DMEM medium containing $1 \mathrm{~g} / \mathrm{L}$ penicillin-streptomycin (Invitrogen, USA). After confluence, the cultured cells were detached using $2.5 \mathrm{~g} / \mathrm{L}$ trypsin (Invitrogen, USA), seeding at the density of $3 \times 10^{4} / \mathrm{cm}^{2}$.

\section{Real-time PCR}

$5 \times 10^{6}$ chondrocytes of the second passage were incubated in respective solutions with different osmolarities for further $2 \mathrm{~h}$. Total RNA was extracted from chondrocytes with Total RNA kit (Omega, USA). The first strand complementary DNA (cDNA) was synthesized from $1 \mu \mathrm{g}$ isolated mRNA yields using reverse transcription kit (TOYOBO: FSQ-201, JAPAN) and used as templates for real-time PCR. The expression of mRNA yields was determined quantitatively using SYBR Green mix (TOYOBO: QPS-201, JAPAN) with Bio-Rad qPCR (Bio-Rad Laboratory, USA) instrument. $\beta$-actin was housekeeping gene, and primer's sequences of the targeted genes were listed (Table 1).

The samples underwent 40 cycles consisting of the following steps: denaturation at $94^{\circ} \mathrm{C}$ for $20 \mathrm{~s}$, annealing at $65^{\circ} \mathrm{C}$ for $20 \mathrm{~s}$, and extension at $72^{\circ} \mathrm{C}$ for $30 \mathrm{~s}$. Data was analyzed by the use of $\Delta \Delta \mathrm{Ct}$, and results were expressed as $2-\Delta \Delta \mathrm{Ct}$.

\section{Statistical analysis}

All statistical analyses were performed using SPSS version 19.0, and data was expressed as $\bar{x} \pm s$. Analyses of variance (ANOVA) were conducted to compare trends among

Table 1 Primer's sequences of the targeted genes used for real-time PCR

\begin{tabular}{lll}
\hline Target gene & Orientation & Primer sequence (5'-3') \\
\hline$\beta$-actin & Forward & CACCATTGGCAATGAGCGGTC \\
& Reverse & AGGTCTITG CGGATGTCCACGT \\
HIF-1a & Forward & TATGAGCCAGAAGAACTITTAGGC \\
& Reverse & CACCTCTITTGGCAAGCATCCTG \\
Collagen II & Forward & CCTGGCAAAGATGGTGAGACAG \\
& Reverse & CCTGGTITCCACCTTCACCTG \\
\hline
\end{tabular}


irrigation solutions with different osmolarities, and Student's $t$ test was conducted to compare trends between saline and balanced salt groups, with the level of significance set at $p<0.05$.

\section{Results}

Cell death in cartilage mechanical injury model

The mechanical injury model demonstrated that in situ dead chondrocytes were mainly localized to the superficial zone orientated tangentially to the articular surface (Figure $2 \mathrm{a}-\mathrm{h}$ ) with the change of medium osmolarity. The percentage of cell death was gradually decreased with the increasing of osmolarity in the superficial zone (saline: $P=0.001$; balanced salt: $P=0.001$ ) (Figure $2 \mathrm{i}, \mathrm{j}$ ). There was no statistically significant difference of cell death in deep zone of articular cartilage (saline: $P=0.93$; balanced salt: $P=0.92$ ) (Figure $2 \mathrm{k}, \mathrm{l}$ ). Compared with the saline of primary solutions, the dead cells in the superficial
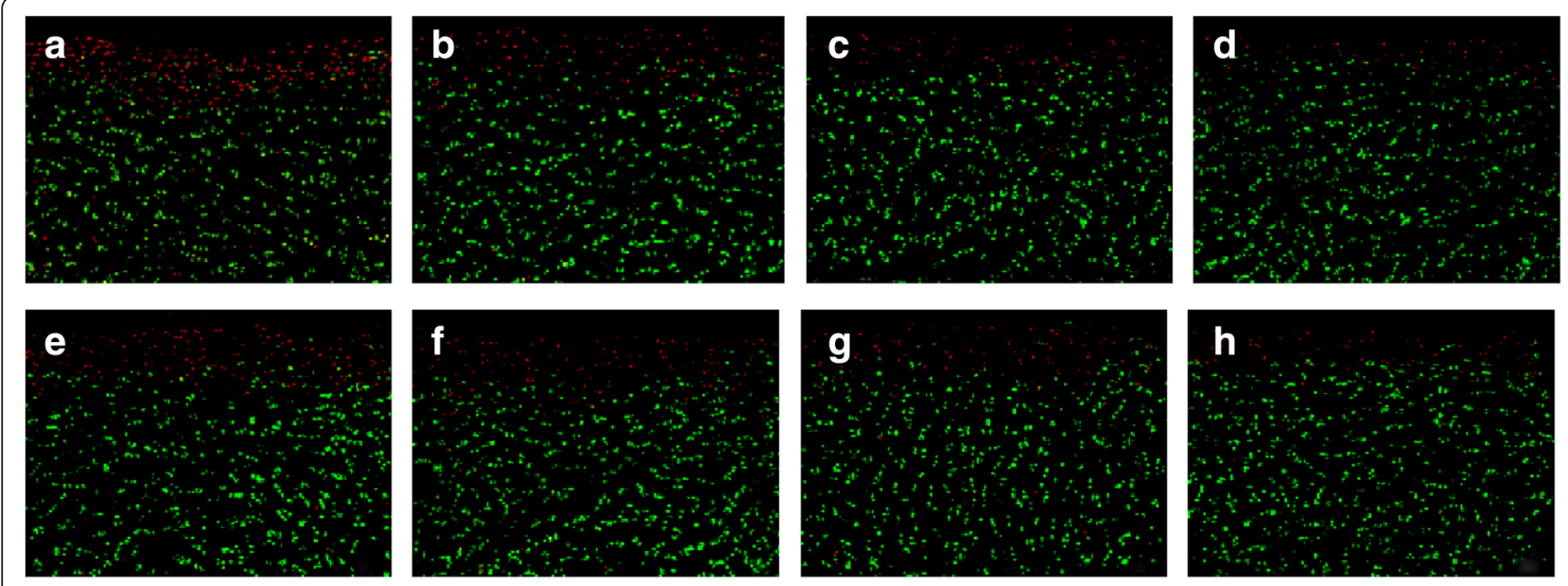

i

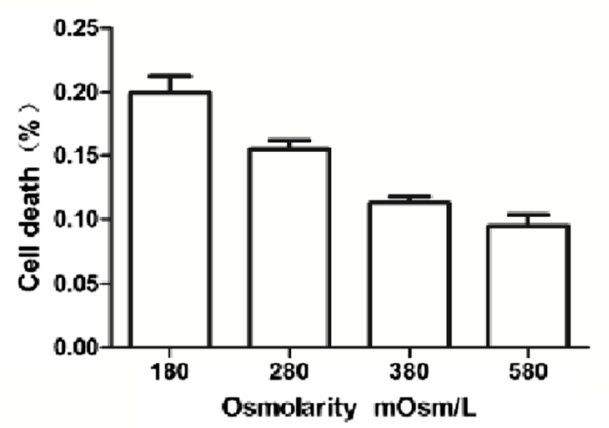

j

$\mathbf{k}$

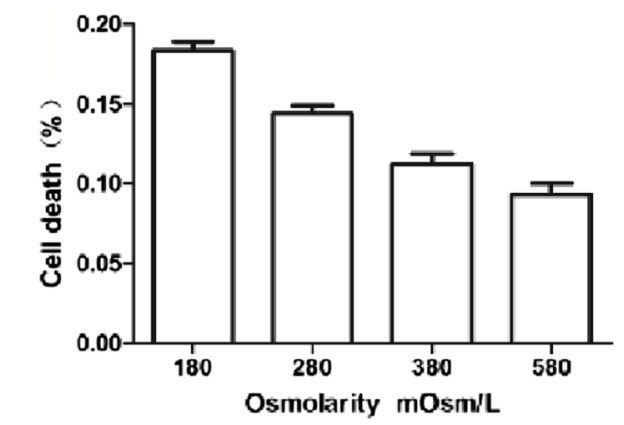

I
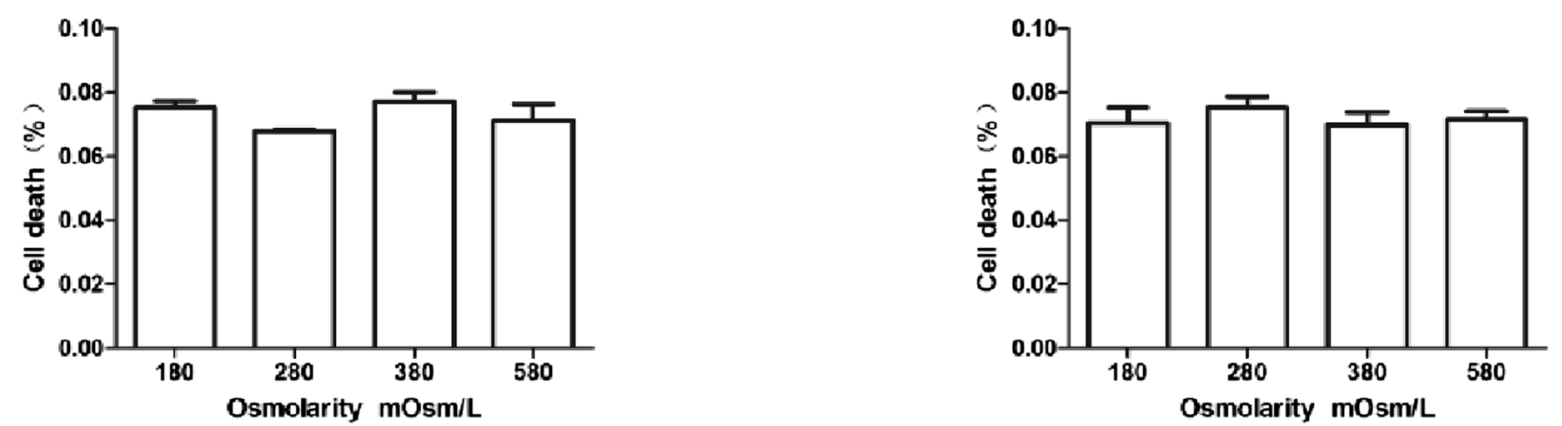

Figure 2 Effect of different osmolarity irrigation solutions on cell viability and cell death. Using laser confocal microscopy to observe the survival of the chondrocytes, red stands for dead cells, green stands for living cells (a-d) saline; (e-h) balanced salt; (a, e) 180 mOsm/L; (b, f) $280 \mathrm{mOsm} / \mathrm{L} ; \mathbf{( c , ~ g ) ~} 380 \mathrm{mOsm} / \mathrm{L}$; and $\mathbf{( d ,} \mathbf{h}) 580 \mathrm{mOsm} / \mathrm{L}$. Effects of different osmolarity irrigation solutions on cell death in the superficial or deep zone. (i, k) Saline; (j, I) balanced salt; (i, j) superficial zone; and (k, I) deep zone ( $n=6$ for each osmolarity; ANOVA tests for superficial zone: saline: $P=0.001$; balanced salt: $P=0.001$; deep zone: saline: $P=0.93$; balanced salt: $P=0.92$ ). 
zone were statistically significantly lower $(P=0.001)$ in the balanced salt (Figure $3 \mathrm{a}-\mathrm{c}$ ); however, there was no statistically significant difference $(P=0.24)$ in the deep zone cells (Figure 3d).

\section{Chondrocyte apoptosis in cartilage}

The chondrocyte apoptosis detected by TUNEL assay was shown that fewer positive cells were observed in different osmolarity groups compared with the positive control (Figure $4 \mathrm{a}-\mathrm{f}$ ). Less than 1\% of chondrocytes were positive in apoptotic assay of different osmolarity groups after $2 \mathrm{~h}$. The apoptosis indices in different osmolarities $(180,280,380$, and $580 \mathrm{mOsm} / \mathrm{L})$ were $0.73 \%$, $0.72 \%, 0.65 \%$, and $0.64 \%$, respectively, and there was no statistically significant difference among them $(P=0.158)$ (Figure 4g).

\section{Proteoglycan elution}

Considerable amounts of proteoglycan were washed out from the cartilage matrix for explants exposed to the different osmolarity medium. It was noted that irrigation solutions of osmolarity $180 \mathrm{mOsm} / \mathrm{L}$ extracted far more proteoglycan from the cartilage matrix than other osmolarity medium, no matter in the saline or the balance salt solutions. With increasing of the osmolarity, the total contents of proteoglycan elution were gradually decreased (saline: $P=0.002$; balanced salt: $P=0.001$ ) (Figure $5 \mathrm{a}, \mathrm{b}$ ), where there was no statistically significant difference between 180 and $280 \mathrm{mOsm} / \mathrm{L}$ (saline: $P=0.39$; balanced salt: $P=0.64)$. Compared with the saline of primary solutions, proteoglycan elution was statistically significantly decreased $(P=0.045)$ in balanced salt solutions (Figure 5c).

\section{Expression of HIF-1a and type II collagen mRNA in chondrocytes}

After incubation of chondrocytes in different solutions (saline, balanced salt), along with increasing of the osmolarity, HIF-1 $\alpha$ and type II collagen mRNA yields were the least for chondrocytes exposed to $180 \mathrm{mOsm} / \mathrm{L}$ medium and were the greatest for chondrocytes exposed to $380 \mathrm{mOsm} / \mathrm{L}$ medium and then decreased with increasing of osmolarity (saline: $P=0.001$; balanced salt: $P=0.001$ ) (Figure 6a, b; Figure 7a, b). However, there was no statistically significant difference between the 180 and $280 \mathrm{mOsm} / \mathrm{L}$ groups (saline: $P=0.058$; balanced salt: $P=0.087)$. HIF- $1 \alpha(P=0.017)$ and type II collagen mRNA $(P=0.034)$ yields from the chondrocytes of the balanced salt solutions were statistically significantly more than the saline (Figures 6c and 7c).

\section{Discussion}

Sharp articular cartilage injury with a scalpel is a reproducible model in vitro, which allows spatial quantification of
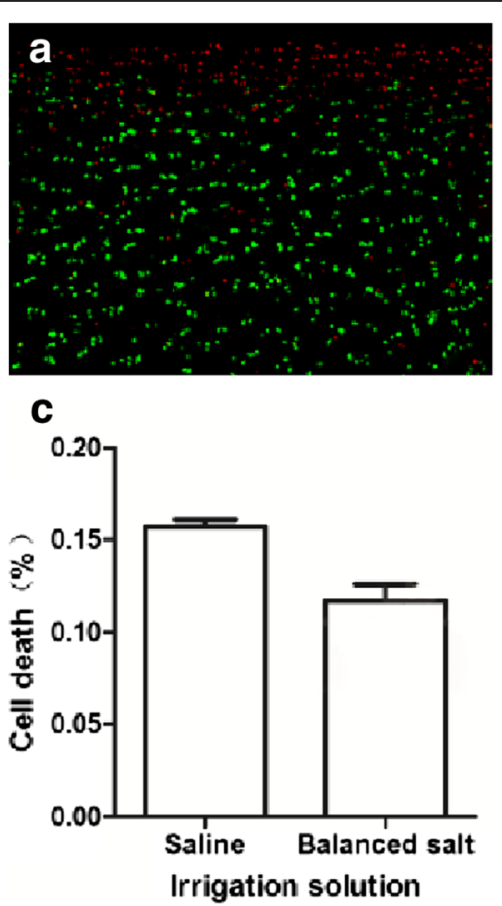

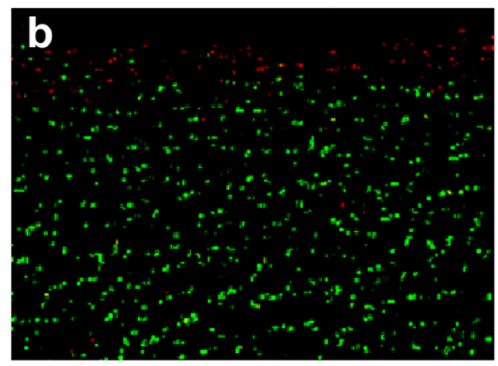

d

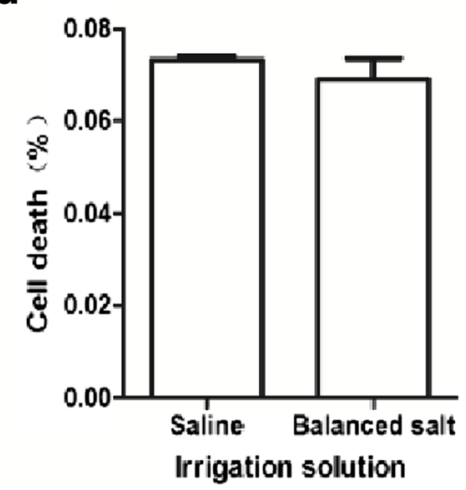

Figure 3 Effect of different primary solutions on cell death in superficial and deep zone. In the human articular cartilage mechanical injury model (a) saline (superficial zone); (b) balanced salt (superficial zone); the percentage of cell death in balanced salt and saline solutions (c) superficial zone; and $(\mathbf{d})$ deep zone $(n=6$ for each solution; $t$ tests for superficial zone: $P=0.001$; deep zone: $P=0.24$ ). 

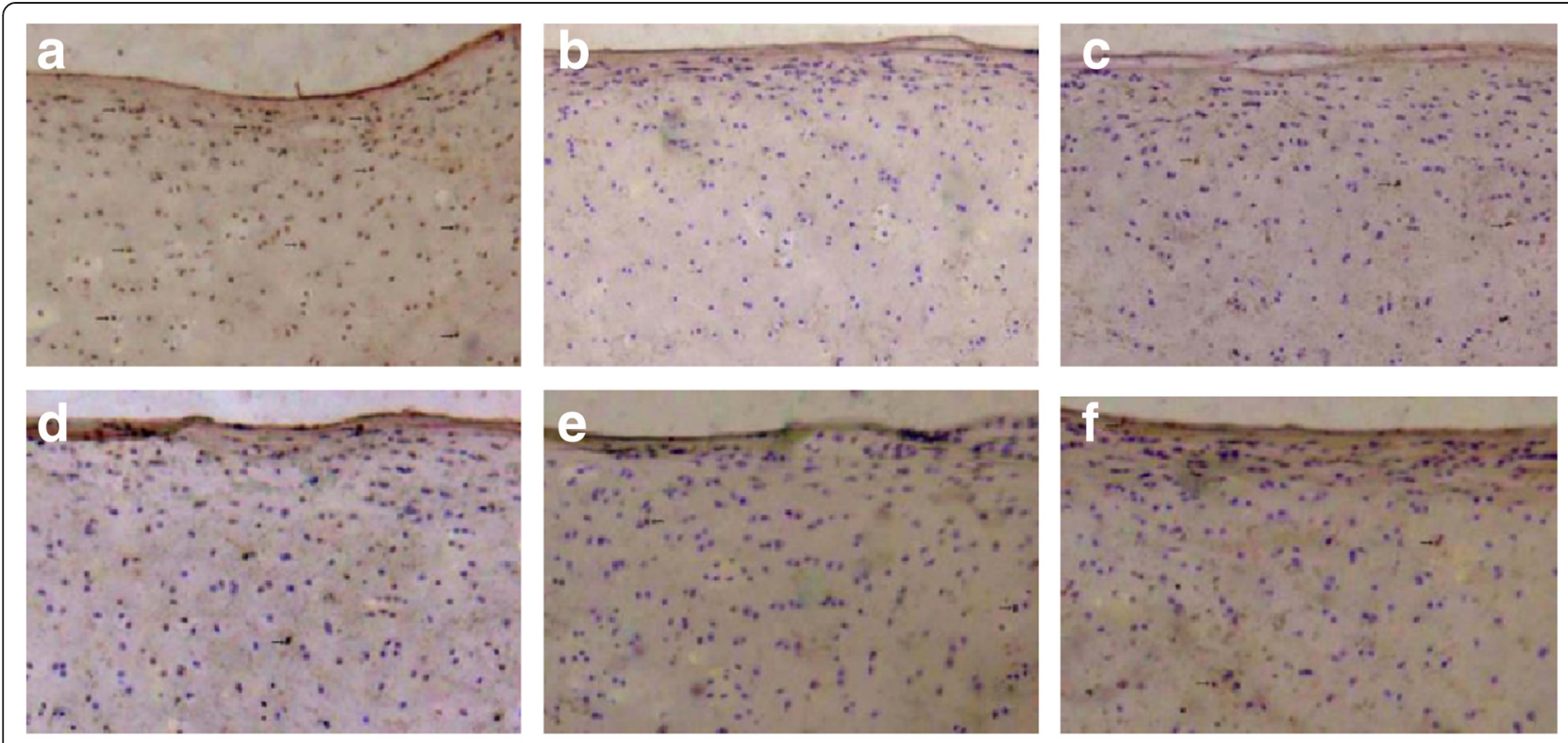

\section{g}

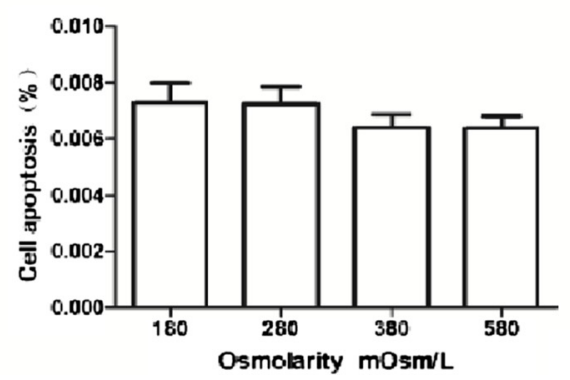

Figure 4 Effect of different osmolarities of saline on chondrocytes apoptosis and representative sections from TUNEL assay. In the human articular cartilage mechanical injury model, sepia stands for apoptotic cells (a) positive control; (b) negative control; (c) $180 \mathrm{mOsm} / \mathrm{L}$; (d) $280 \mathrm{mOsm} / \mathrm{L}$; (e) $380 \mathrm{mOsm} / \mathrm{L}$; (f) $580 \mathrm{mOsm} / \mathrm{L}$; and (g) the percentage of apoptotic cells ( $n=6$ for each osmolarity, ANOVA test, $P=0.158)$.
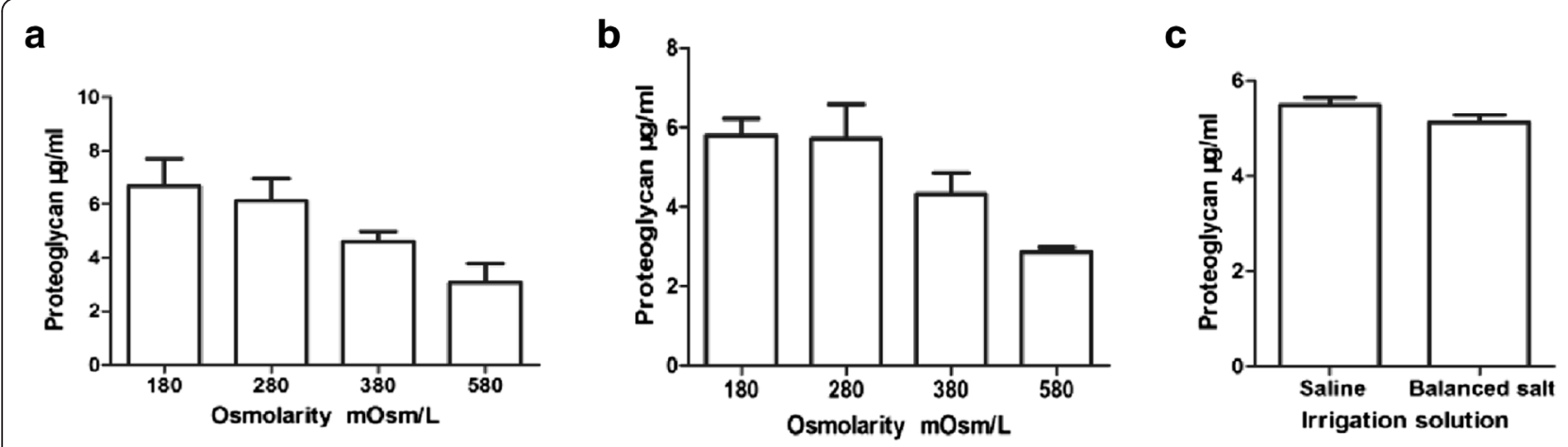

Figure 5 Effect of different osmolarity irrigation solutions on proteoglycan elution. In the human articular cartilage mechanical injury model (a) saline in different osmolarities; (b) balanced salt in different osmolarities; and (c) comparison of proteoglycan elution between the saline and balanced salt. ( $n=6$ for each osmolarity and solution; ANOVA tests for saline: $P=0.002$; balanced salt: $P=0.001$; $t$ test of saline vs balanced salt: $P=0.045)$. 

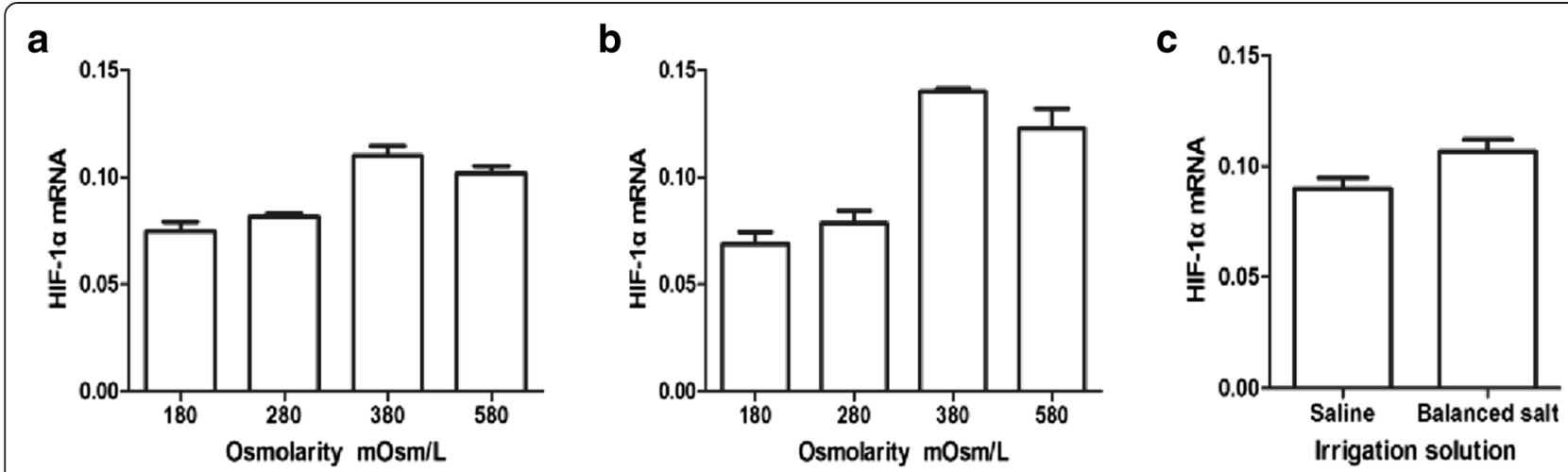

Figure 6 Effect of different osmolarity irrigation solutions on expression of HIF-1a mRNA. In the human articular chondrocytes with real-time PCR (a) saline in different osmolarities; (b) balanced salt in different osmolarities; and (c) comparison of HIF-1a mRNA between saline and balanced salt (ANOVA tests for saline: $P=0.001$; balanced salt: $P=0.001 ; t$ test for saline vs balanced salt: $P=0.017, n=3$ for each osmolarity and solution; repeat for three times, the figure shows only one representative).

in situ chondrocyte death within all zones of the injured cartilage to predict the responses of sharply wounded articular cartilage during articular surgery $[3,10]$. Scalpel-cut explants are as models to study the effect of medium osmolarity on chondrocyte death for the reason of effectively avoiding the variation in matrix damage and chondrocyte death [11]. The duration of explant exposed to a medium with altered osmolarity (about $2 \mathrm{~h}$ ) was simulated to the time during arthroscopic surgery. Amin et al. proved that there was no increase in chondrocyte death from $2.5 \mathrm{~h}$ to 7 days in animal model [12], suggesting that early use of irrigation solutions (about $2 \mathrm{~h}$ ) played an important role in chondrocyte survival. The integrity of superficial zone and survival of in situ chondrocytes are significantly important for maintaining the biomechanical properties of cartilage and preventing cartilage degeneration [1]. Our findings show that chondrocytes in the superficial zone are the most susceptible to the fullthickness cartilage injury and dead cells of higher density are mainly localized to the superficial zone of the scalpel-cut edge. The results show similar trends with Amin et al.'s reports in human articular cartilage [6]; moreover, we further tested effects of osmolarity on chondrocyte survival after mechanical injury in different irrigation solutions with osmolarity gradients, elucidated the mechanism with detecting apoptosis index, exploring the influences of osmolarity on cartilage matrix proteoglycan elution and in vitro chondrocyte metabolism.

The accurate mechanism responsible for the impact of the medium osmolarity on the chondrocyte survival following mechanical injury remains unclear. Bush et al. pointed out that the volume change of chondrocytes exposed to the mechanical stress had a significant effect on cell survival [13]. Chondrocyte morphology and metabolism was determined in the cultured osmolarity medium of $280 \sim 580 \mathrm{mOsm} / \mathrm{L}$ in vitro, Xu et al. found that the cell volume swelled $20 \%$ in $280 \mathrm{mOsm} / \mathrm{L}$ and shrinked $15 \%$ in $580 \mathrm{mOsm} / \mathrm{L}$ [10]. Hyposmolarity makes cell swelling and hyperosmolarity makes cell shrinkage, by

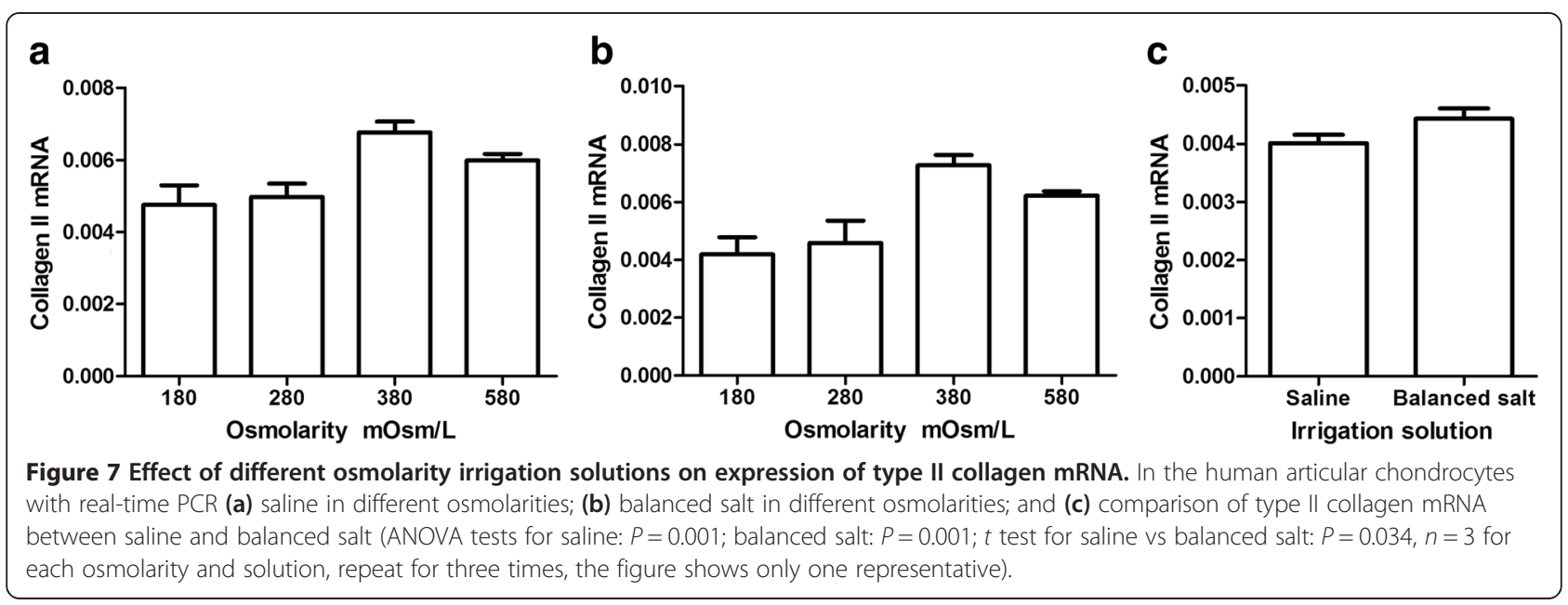


which swelling chondrocytes of osmotic responses become more susceptible to mechanical injury [14]. Those cell death induced by mechanical injury may be the result of necrosis, apoptosis, or other forms. Our data demonstrated that less than $1 \%$ of these chondrocytes were positive in apoptosis assay in different osmolarity groups, which indicated that the cell death in the cartilage following mechanical injury was mainly not caused by apoptosis.

The elastic component is decreased due to the proteoglycan elution, affecting the structure of cartilage, where the viability of chondrocytes is more susceptible to mechanical injury. Proteoglycan is an important component in the cartilage matrix accounting for about $7 \%-10 \%$ of the cartilage that plays an important role in stress load dispersion in articular cartilage. The matrix proteoglycan can easily diffuse through the intact cartilage surface and appear in the incubation medium under certain conditions $[14,15]$. The extracellular osmolarity is greater with precise values determined by the proteoglycan concentration when proteoglycan is released from the collagen meshwork to the medium [16,17]. Our study finds that amounts of proteoglycan are washed out from the cartilage matrix and proteoglycan elution decreases with the increasing medium osmolarity. Reduction of proteoglycan in cartilage matrix can affect the interaction between the extracellular matrix and chondrocytes, which produce a great impact on cell survival. The elution of proteoglycan will be expected to occur primarily from the superficial zone of the cartilage because the water content is the highest and the permeability is superior in the superficial areas [18]. The osmotic responses of chondrocytes may have been exacerbated by the disruption to the extracellular matrix from the scalpel wound where the support structure of the collagen fibers is damaged and the extraction of proteoglycan is accelerated, which is the indirect cause of the chondrocyte death [19].

Chondrocyte metabolism is inordinately inhibited in different osmolarity medium, which may affect cartilage repair. Through the regulation of osmolarity channels, chondrocytes maintain optimal volume and hence mediate any changes in cell metabolism and biosynthesis following exposure to osmotic challenge [20]. As the specific genes in chondrocytes, HIF- $1 \alpha$ and type II collagen indicate the metabolic level of chondrocytes. Chondrocytes can be stimulated in hypoxia environment via HIF-1 $\alpha$ to express numerous genes associated with chondrocyte anabolism and differentiation, including Sox9, TGF- $\beta$, etc., and hypoxia and HIF-1 $\alpha$ are associated with type II collagen production [21-23]. The present study indicates that HIF- $1 \alpha$ and type II collagen mRNA yields in the primary cultured chondrocytes are the least in $180 \mathrm{mOsm} / \mathrm{L}$ medium and are the greatest in $380 \mathrm{mOsm} / \mathrm{L}$, the later closing to the physiological osmolarity of the synovial fluid. Negoro et al. indicated that chondrocytes cultured in closed physiological osmolarity solutions had stronger ability of proteoglycan synthesis in the three-dimensional culture of primary chondrocytes [24].

Saline may not be beneficial to cartilage repair after injury. The cell death in superficial zone, proteoglycan elution, and inhibition of HIF- $1 \alpha$ and collagen II expression were significantly less in the balanced salt solutions. By detecting ${ }^{35} \mathrm{SO} 4$ uptake rate, Gulihar et al. compared proteoglycan synthesis levels of chondrocyte in different fluids, finding that Ringer's lactate inhibited cartilage metabolism by $10 \%$, whereas saline caused $35 \%$ inhibition [13]. There will be an additional increase in the extracellular sodium ion concentration by the use of saline, potentially leading to altered activity of sodiumdependent membrane transporters, particularly those involved in chondrocyte volume and metabolism [22]. In addition to sodium ion, balanced salt also contains potassium and magnesium ion, etc., which probably supports chondrocyte metabolism better than $0.9 \%$ saline that is considered to be not physiological.

Our research still had certain limitations. Firstly, what we chose was the cartilage tissue from knee arthroplasty, but not normal cartilage tissue, which was not easy to obtain. Secondly, the mechanical injury model was steerable, but the real surgical instruments injury was much more intricate. So the influence of osmolarity of irrigation solutions on cartilage repair after injury should be further examined in an in vivo model or in a clinical setting. Finally, it needed to further investigate the accurate molecular mechanism, such as the expression and interaction of putative potential osmotic channels (TRPV4 and BKCa) in chondrocytes in different osmolarities [25,26].

\section{Conclusions}

The osmolarity of irrigation solutions plays an important role in the survival and metabolic state of chondrocytes following mechanical injury, and the chondrocyte death is not caused by apoptosis. Increasing osmolarity of irrigation solutions may be chondroprotective with decreasing the chondrocyte death, reducing inhibition of metabolism and proteoglycan elution, ultimately preventing cartilage degeneration and promoting integrative repair.

\section{Competing interests}

The authors declare that they have no competing interests.

\section{Authors' contributions}

HY carried out the laser confocal microscopy and drafted the manuscript; ZY participated in the real-time PCR, performed the statistical analysis, and participated in the manuscript preparation. DX determined the proteoglycan elution and TUNEL assay; LS participated in the specimen collection; ST conceived of the study and supervised its design and completed the manuscript. All authors read and approved the final manuscript. 


\section{Authors' information}

Dr. Sun is the specialist of sports medicine and arthroscopy, and the main research fields are osteoarthritis and cartilage.

\section{Acknowledgements}

The authors give thanks to Dr. He Qihua for his confocal microscope expertise and Peking University Health Science Center for offering the confocal microscope measurement. The project was funded and supported by the Peking University People's Hospital, Beijing.

\section{Author details}

${ }^{1}$ Arthritis Clinic and Research Center, Peking University People's Hospital, No. 11 Xizhimen South Street, Xicheng District, Beijing 100044, China. ${ }^{2}$ Clinic Molecular Institute, Peking University People's Hospital, No. 11 Xizhimen South Street, Xicheng District, Beijing 100044, China.

Received: 12 July 2014 Accepted: 8 January 2015

Published online: 28 January 2015

\section{References}

1. Hunziker EB. Articular cartilage repair: basic science and clinical progress. A review of the current status and prospects. Osteoarthritis Cartilage. 2002;10:432-63.

2. Bertram KL, Krawetz RJ. Osmolarity regulates chondrogenic differentiation potential of synovial fluid derived mesenchymal progenitor cells. Biochem Biophys Res Commun. 2012;42:455-61.

3. Bush PG, Hall AC. The osmotic sensitivity of isolated and in situ bovine articular chondrocytes. J Orthop Res. 2001;19:768-78.

4. Bush PG, Hall AC. Passive osmotic properties of in situ human articular chondrocytes within non-degenerate and degenerate cartilage. J Cell Physiol. 2005;204:309-19.

5. Amin AK, Huntley JS, Simpson A, Hall AC. Increasing the osmolarity of joint irrigation solutions may avoid injury to cartilage: a pilot study. Clin Orthop. 2010;468:875-84

6. Amin AK, Huntley JS, Patton JT, Brenkel IJ. Hyperosmolarity protects chondrocytes from mechanical injury in human articular cartilage: an experimental report. J Bone Joint Surg Br. 2011;93:277-84

7. Mainil P, Aigner T, Brittberg M. Histological assessment of cartilage repair: a report by the Histology Endpoint Committee of the International Cartilage Repair Society (ICRS). J Bone Joint Surg Am. 2005:85:45-57.

8. Poole CA, Brookes NH, Clover GM. Confocal imaging of the human keratocyte network using the vital dye 5-chloromethylfluorescein diacetate. Clin Experiment Ophthalmol. 2003;31:147-54.

9. Nakashima K, Yamashita K, Kenichi K. An in vitro model of cartilage degradation by chondrocytes in a three-dimensional culture system. Int J Biomed Sci. 2012;8:249-57.

10. Xu X, Urban JP, Tirlapur UK. Osmolarity effects on bovine articular chondrocytes during three-dimensional culture in alginate beads. Osteoarthritis Cartilage. 2010;18:433-9.

11. Huntley JS, Bush PG, McBirnie JM, Simpson AH, Hall AC. Chondrocyte death associated with human femoral osteochondral harvest as performed for mosaicplasty. J Bone Joint Surg Am. 2005;87:351-60.

12. Amin AK, Huntley JS, Bush PG, Simpson AH, Hall AC. Osmolarity influences chondrocyte death in wounded articular cartilage. J Bone Joint Surg Am. 2008;90:1531-42.

13. Bush PG, Hodkinson PD, Hamilton GL, Hall AC. Viability and volume of in situ bovine articular chondrocytes: changes following a single impact and effects of medium osmolarity. Osteoarthritis Cartilage. 2005;13:54-65.

14. Gulihar A, Bryson DJ, Taylor GJ. Effect of different irrigation fluids on human articular cartilage: an in vitro study. Arthroscopy. 2013;29:251-6.

15. Reiner $G$, Jurka $T$, Rainer J. Influence of various irrigation fluids on articular cartilage. Arthroscopy. 1995;11:263-9.

16. Urban JP, Hall AC, Gehl KA. Regulation of matrix synthesis rates by the ionic and osmotic environment of articular chondrocytes. J Cell Physiol. 1993;15:262-70

17. Comper WD, Williams RP. Osmotic flow caused by chondroitin sulfate proteoglycan across well-defined nuclepore membranes. Biophys Chem. 1990;36:215-22.

18. Xu JW, Zaporojan V, Peretti GM. Injectable tissue-engineered cartilage with different chondrocyte sources. Plast Reconstr Surg. 2004;113:1361-71.
19. Torzilli PA, Grigiene R, Borrelli J, Helfet DL. Effect of impact load on articular cartilage: cell metabolism and viability, and matrix water content. J Biomech Eng. 1999;121:433-41.

20. Bush $P G$, Hall AC. Regulatory volume decrease (RVD) by isolated and in situ bovine articular chondrocytes. J Cell Physiol. 2001;187:304-14.

21. Coimbra IB, Jimenez SA, Hawkins DF. Hypoxia inducible factor-1 alpha expressionin human normal and osteoarthritic chondrocytes. Osteoarthritis Cartilage. 2004;12:336-45.

22. Brucker PU, Izzo NJ, Chu CR. Tonic activation of hypoxia-inducible factor 1alpha in avascular articular cartilage and implications for metabolic homeostasis. Arthritis Rheum. 2005;52:3181-91.

23. McAlinden A, Haag J, Bau B, Gebhard PM, Aigner T. Quantification of mRNA expression levels in articular chondrocytes with PCR technologies. Methods Mol Med. 2004;100:79-100.

24. Negoro K, Kobayashi S, Takeno K. Effect of osmolarity on glycosaminoglycan productionand cell metabolism of articular chondrocyteunder three dimensional culturesystem. Clin Exp Rheumatol. 2008;26:534-41.

25. Mimi N, Holly A, Bartholomew J, Dana S. Functional characterization of TRPV4 as an osmotically sensitive ion channel in porcine articular chondrocytes. Arthritis Rheum. 2009;60:3028-37.

26. Liedtke WB. TRPV4 plays an evolutionary conserved role in the transduction of osmotic and mechanical stimuli in live animals. J Physiol. 2005;56:53-8.

\section{Submit your next manuscript to BioMed Central and take full advantage of:}

- Convenient online submission

- Thorough peer review

- No space constraints or color figure charges

- Immediate publication on acceptance

- Inclusion in PubMed, CAS, Scopus and Google Scholar

- Research which is freely available for redistribution 